Trauma Berufskrankh 2016 · [Suppl 3]: 18:S246-S253

DOI 10.1007/s10039-015-0088-3

Online publiziert: 14. Oktober 2015

๑) Springer-Verlag Berlin Heidelberg 2015

CrossMark

Karen Piefel • Thomas Schneider • Michael Heinrich Seegenschmiedt

Strahlenzentrum Hamburg, Hamburg, Deutschland

\title{
Strahlentherapie bei Fibromatosen
}

\section{Ergebnisse bei Morbus Dupuytren und Morbus Ledderhose}

matosen der Handflächen (MD) und der Fußsohlen (ML) sowie die Arthrofibrosen und der Morbus Peyronie gehören.

\section{Strahlenbiologische Rationale}

Ähnlich wie bei der Entwicklung von Keloiden, wo überschießendes Wachstum von Narbenbindegewebe im Rahmen der Wundheilung auftritt, entwickelt sich beim MD und ML eine Hyperproliferation von Bindegewebe in der Palmar- bzw. Plantaraponeurose. Im pathophysiologischen Ablauf ist zu beobachten, dass dabei Kollagen Typ III durch Kollagen Typ I ersetzt wird, was die normale Wundheilung stören kann. Je nach Ablauf der Vorgänge bildet sich mehr TypIII („early type“) oder Typ-I („late type“)Kollagen. Eine derartige Störung der physiologischen Zellproliferation wird auch für die Bildung der hyperproliferativen Gewebe der Handflächen und Fußsohlen bei MD/ML vermutet; dies kann primär aus unbekannten Gründen oder sekundär durch Trauma oder iatrogene Auslösemechanismen, wie z. B. eine chronische Hypoxie, induziert werden. Histologisch findet man in den Knoten und Strängen atypische Fibroblasten, Myofibroblasten mit Ablagerung extrazellulärer Matrixkomponenten, vor allem Kollagen, Fibronectin, Elastin und Proteoglykane. Die Knoten und Stränge bilden sich in den tiefen Strukturen der Palmar- oder Plantarfaszie.

Die strahlenbiologische Rationale für die Anwendung ionisierender Strahlen bei MD/ML zur nachhaltigen Beeinflussung des Krankheitsverlaufs ergibt sich aus der Radiosensitivität von bestimmten Zielzellen und biologischen Mechanismen, die günstig zu beeinflussen sind: $\mathrm{Zy}$ tokin „tissue growth factor $\beta$ “ (TGF- $\beta$ ) sowie die Proliferation der Myofibroblasten (MFb) Typ I bis Typ III, die nach der Bestrahlung durch die Ausbildung von postmitotischen Myofibroblasten (pMFb) völlig zum Erliegen kommt (• Abb. 1).

Die - Abb. 1 stellt schematisch die Zielzellen und Mechanismen der Radiosensitivität der hyperproliferativen Weichgewebe bei der Fibrosebildung in einem Lungenmodell dar. Das Schlüsselmolekül ist TGF- $\beta$, der die Umwandlung von Vor-

\begin{tabular}{|ll}
\hline Abkürzungsverzeichnis \\
\hline CTC & Common Toxicity Criteria \\
\hline DEGRO & $\begin{array}{l}\text { Deutsche Gesellschaft für } \\
\text { Radioonkologie }\end{array}$ \\
\hline H-CTX & Handchirurgie \\
\hline ICD-10-GM & $\begin{array}{l}\text { International Classification of } \\
\text { Diseases and Related Health } \\
\text { Problems, 10. Revision, Ger- } \\
\text { man Modifikation }\end{array}$ \\
\hline LENT & $\begin{array}{l}\text { Late Effects Normal } \\
\text { Tissue (Score für } \\
\text { Spätnebenwirkungen) }\end{array}$ \\
\hline$M D$ & Morbus Dupuytren \\
\hline$M L$ & Morbus Ledderhose \\
\hline RT & Radiotherapie \\
\hline S2e-Leitlinie & $\begin{array}{l}\text { Leitlinie einer wissenschaft- } \\
\text { lichen Fachgruppe }\end{array}$ \\
\hline TGF- $\beta$ & \begin{tabular}{l} 
Transforming growth factor $\beta$ \\
\hline
\end{tabular} \\
\hline
\end{tabular}



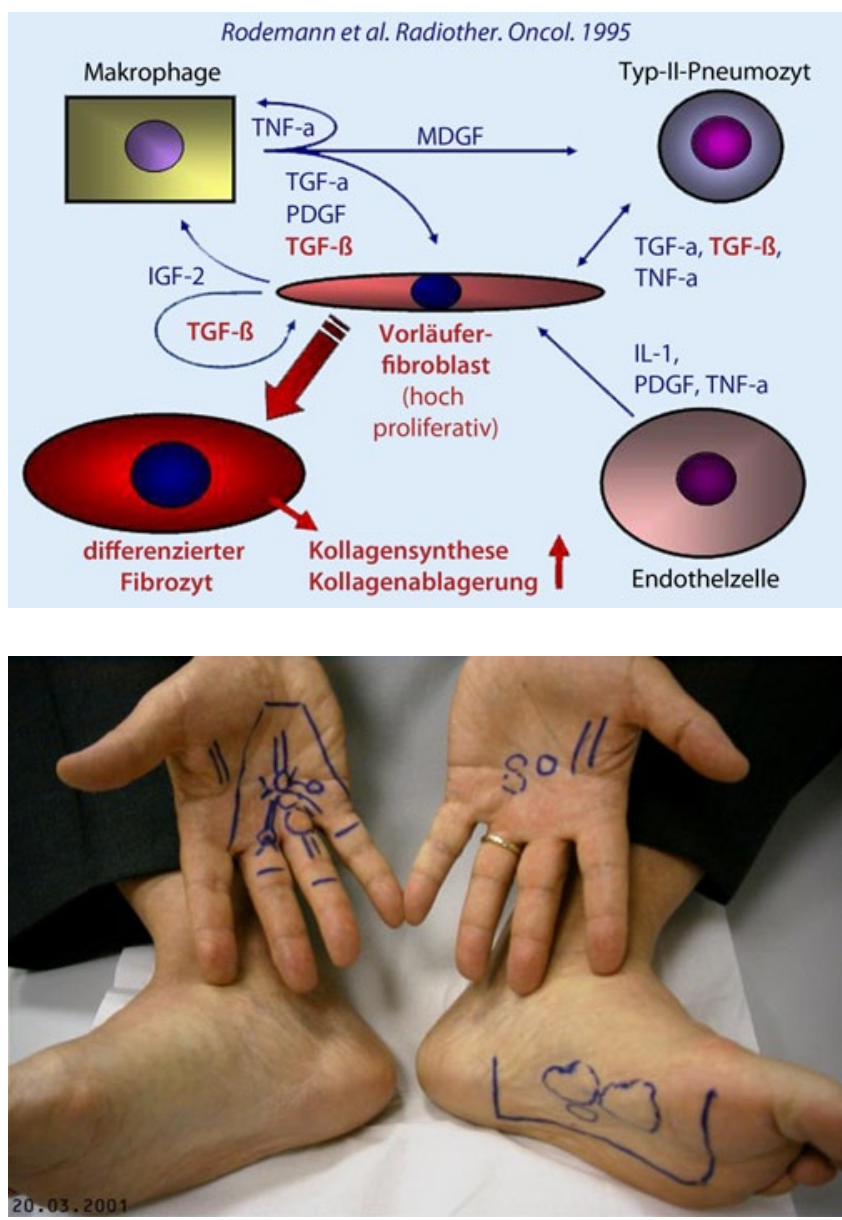

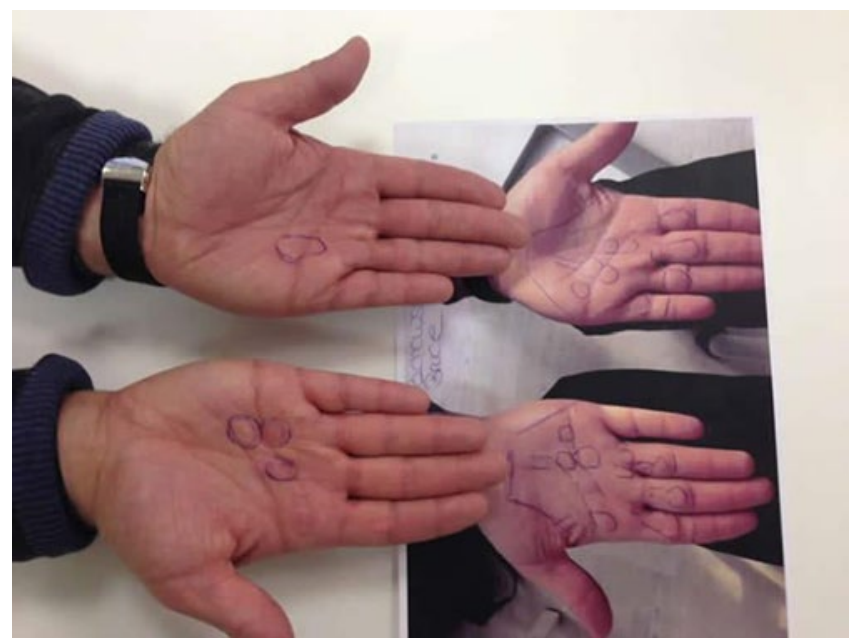

Abb. $1<$ Zielzellen und Mechanismen der Strahlenempfindlichkeit hyperproliferativer Weichgewebe bei der Fibrosebildung. ([2], mit freundl. Genehmigung von Elsevier)

Abb. $2<46$-jähriger Mann (positive Familienanamnese, beidseits Morbus Dupuytren, einseitiger Morbus Ledderhose linker Fuß); Indikation zur Strahlentherapie für die rechte Hand und den symptomatischen linken Fuß gegeben. Wait-and-see-Option für linke Hand. (Mit freundl. Genehmigung von Prof. Dr. M.H. Seegenschmiedt)

Abb. $3<$ Nachgewiesener Progress des Morbus Dupuytren (MD) von Stadium N [Hände rechts, keine Strahlentherapie (RT)Indikation] zu einem Stadium N/I (Hände links, jetzt mit RT-Indikation) im 12-Monats-Follow-up. (Mit freundl. Genehmigung von Prof. Dr. M.H. Seegenschmiedt)

läuferfibroblasten $\mathrm{zu}$ entdifferenzierten Fibrozyten steuert und für die Narbenbildung verantwortlich ist. Dieser Wachstumsfaktor und die durch ihn induzierten Mechanismen sind aber auch empfindlich gegenüber ionisierender Strahlung.

\section{Klinische Rationale}

MD/ML verlaufen in 3 Phasen, die unterschiedlich auf ionisierende Strahlen reagieren:

1. radiosensitive, hyperproliferative Phase mit einer erhöhten Anzahl von Fibroblasten, von Knoten und beginnende Strangbildung,
2. weniger radiosensitive Involutionsphase mit erhöhter Anzahl von Myofibroblasten in Faserbündeln, die zu vermehrten Strängen und beginnenden Kontrakturen führen, sowie

3. radioresistente Endphase mit kollagenen Fasern und dominierendem Bindegewebe, das zellarm ist und von starker Narbenbildung mit Kontrakturen charakterisiert ist.

Die Erkrankung kann langsam fortschreiten oder über Jahre stabil bleiben; nur selten kommt es zur Spontanremission. Ohne Therapie schreiten MD/ML bei etwa $50 \%$ der Betroffenen innerhalb von 5 Jahren weiter voran [3].

Beim ML werden Knoten und Stränge selten schon in der Frühphase festgestellt; meist alarmieren Dysfunktion (Gangstörung) oder Symptome (Schmerzen, Spannung, Druck, Juckreiz) den Patienten und führen zur ersten Vorstellung beim Arzt. Das zusätzliche Auftreten von Knöchelpolstern oder einer Penisverkrümmung (Morbus Peyronie) bei Männern erhärtet die Diagnose [4]. Der klinische Verlauf von MD/ML hängt von vielen individuellen Faktoren ab [5]. Eine spontane Remission kann durch Phasen von Progress und Stagnation abgelöst werden; ansonsten kann es auch innerhalb kurzer Zeit zum raschen Voranschreiten einer durch Kontraktur induzierten Dislokation der digitalen Gelenke beim MD oder erheblichen Gangstörungen beim ML kommen. Es gibt auch bestimmte Sonderformen, die durch ihren klinischen Verlauf charakterisiert werden:

- die „milde MD-Variante“ bei Patienten mit Diabetes mellitus und im höheren Alter und

- die „aggressive MD-Variante“ bei jüngeren Menschen mit Ausbruch ab der dritten Lebensdekade, oft mit einem schnellen und beidseitigen Befall verbunden, der sowohl die ulnare als auch die radiale Seite der Handfläche betreffen kann.

\section{Indikation zur Strahlentherapie}

In den letzten Jahren wurde die Bedeutung der Strahlentherapie (RT) in frühen und noch proliferativen Phasen der Krankheit, in der die Operation keine 
Rolle spielt, zunehmend erkannt, da hier strahlenempfindliche Zielzellen vorhanden sind. Deshalb sollte in dieser Phase auch nur bei „progressiver Erkrankung“ die Indikation zur Strahlentherapie gestellt werden. Klinische Zeichen für eine Progression können dabei sein:

- Bildung von einem oder mehreren progressiven Knoten,

- Neubildung von Strängen und/oder

- zunehmendes funktionelles Defizit in einem oder mehreren Fingern bis zu $10-30^{\circ}$ oder

- Übergang von Stadium N zu Stadium N/I.

Patienten in „späteren Stadien“ mit einem Funktionsdefizit $>30^{\circ}$ sind nicht mehr für eine RT qualifiziert. Ein typisches klinisches Beispiel zeigt $\bullet$ Abb. 2:

Die Indikation zur Strahlentherapie folgt also ganz strikt der klinischen Stadieneinteilung, die sich auf den Funktionsverlust der Fingerstreckung beim MD [6] und den Funktionsverlust der Elastizität der Haut der Fußsohle und Tiefenstrukturen des Fußes bei ML ([7]; - Tab. 1 und 2) stützt.

\section{Weitere Behandlungsoptionen}

Trotz jahrzehntelanger Forschung steht derzeit keine kurative Behandlung für den MD/ML zur Verfügung. Alle therapeutischen Bemühungen ( $\bullet$ Tab. 3), einschließlich der lokalen Injektion von Enzymen, Medikamente oder lokale RT für involvierte Bereiche, minimalinvasive oder offene radikale Chirurgie zielen daher darauf ab, das Fortschreiten der Knoten und Stränge zu verhindern oder den beeinträchtigenden Funktionsstatus zu verbessern.

Während die Hand- oder Fußoperation zumeist mit der Verbesserung der Funktionsdefizite gerechtfertigt werden kann, dient die Bestrahlung nur der Verhinderung einer zunehmenden Symptomatik und der Vermeidung von funktionellen Beeinträchtigungen; das Vorhandensein von radiosensitiven Zielzellen und biologischen Mechanismen ist für eine erfolgreiche Interaktion unerlässlich. Der Goldstandard für fortgeschrittene Stadien des MD/ML ist und bleibt die Operation, vor allem wenn Symptome

Trauma Berufskrankh 2016 · [Suppl 3]: 18:S246-S253 DOI 10.1007/s10039-015-0088-3

(c) Springer-Verlag Berlin Heidelberg 2015

\section{K. Piefel · T. Schneider $\cdot$ M.H. Seegenschmiedt}

\section{Strahlentherapie bei Fibromatosen. Ergebnisse bei Morbus Dupuytren und Morbus Ledderhose}

\section{Zusammenfassung}

Hintergrund. Morbus Dupuytren (MD) und Morbus Ledderhose (ML) sind proliferative Bindegewebserkrankungen der Palmar- oder Plantaraponeurose. Zwei Drittel der Patienten weisen eine bilaterale Beteiligung auf. Die Erkrankung ist selten (1-2\% der Bevölkerung), der MD aber häufiger als der ML.

Klinik. Klinisch bestehen im Frühstadium subkutane Knoten und/oder Stränge. In späteren Stadien entstehen Beugekontrakturen der Finger und Funktionsdefizite der Hand beim MD oder Schmerzen mit Gangstörung beim ML.

Therapie und Ergebnisse. Therapieoptionen sind minimalinvasive und invasive operative Maßnahmen, Eigenfetttransplantation, Injektionen von Kollagenase oder Steroiden oder INF-ysowie die Stoßwellentherapie. Eine Operation ist v. a. bei Funktionsstö- rungen (Streckdefizit) der Finger indiziert. Die Strahlentherapie wirkt im Frühstadium der Krankheit vor allem auf die proliferierenden Fibroblasten. Dadurch kann ein Progress bzw. in der Folge eine Operation nach Strahlentherapie in $80-90 \%$ vermieden und eine Remission der Knoten und Stränge in 20-30\% erreicht werden. Dies belegen auch klinische Studien mit fast 500 Patienten und über 5 Jahren Nachbeobachtung. Nach einer Bestrahlung an Hand- oder Fußfläche wurden keine erhöhten Komplikationen im Falle einer noch erforderlichen Operation beobachtet.

Schlüsselwörter

Bindegewebserkrankungen .

Palmaraponeurose · Plantaraponeurose .

Beugekontrakturen · Therapie

\section{Radiation therapy of fibromatosis. Results for Dupuytren's contracture and Ledderhose's disease}

\section{Abstract}

Background. Dupuytren's contracture (MD) and Ledderhose's disease (ML) are connective tissue disorders of palmar and plantar aponeurosis, respectively. Approximately two thirds of patients have a bilateral involvement. Both diseases are rare and occur in only $1-2 \%$ of the population and MD is more frequent than $\mathrm{ML}$ with a predominance in males.

Clinical aspects. Clinical changes in early stages include subcutaneous nodules and/ or cords, while in later stages flexion contractures and functional hand and finger deficits (MD) or pain with gait dysfunction (ML) can occur.

Therapy and results. The most recent treatment options include minimally invasive or invasive hand surgery (aponeurotomy), lipografting, injection of collagenase or steroids, interferon (INF) gamma and local shockwave therapy. Surgery is always indicated in cases of functional impairment. Radiotherapy is effective for early disease stages with proliferating fibroblasts as sensitive target cells. Further progression can be prevented in 80 $90 \%$ of cases and recurrence of nodes and cords in 20-30\% which has been confirmed by clinical studies with almost 500 patients and over 5 years follow-up. The exposure to a 30 Gy radiotherapy dose does not increase the surgical complication rate in case surgery becomes necessary due to a later disease progression.

\section{Keywords}

Connective tissue disorders - Flexion contractures · Palmar aponeurosis · Plantar aponeurosis · Therapy zunehmen und die Funktionen durch die fortschreitende Kontraktur beeinträchtigt werden. Typische chirurgische Eingriffe sind die Durchtrennung der Stränge (Fasziotomie) oder die Exzision von erkrankten Faszienbändern (Fasziektomie) mit oder ohne Entfernung der darüberliegenden Haut. Manchmal sind radikalere Verfahren erforderlich wie die Gesamtfas- ziektomie (Dermatofasziektomie) mit der darüber liegenden Haut. Die Hauptziele der Operation sind die Umkehr der digitalen Kontrakturen und die Hand- bzw. Fußfunktion wieder herzustellen. Eine postoperative Bestrahlung ist in wenigen klinischen Studien beim ML erfolgreich beschrieben worden. 


\begin{tabular}{|c|c|c|c|c|}
\hline RT-Indikation & Stadium & D1 (Daumen) & D2-D5 & Punkte \\
\hline Keine RT-Indikation & 0 & $\begin{array}{l}\text { Weder Knoten noch } \\
\text { Verlust der Abduktion }\end{array}$ & $\begin{array}{l}\text { Kein Streckdefizit } \\
\text { Kein Knoten oder Strang }\end{array}$ & $=0$ \\
\hline Frühe RT-Indikation & $\mathrm{N}$ & $\begin{array}{l}\text { Knoten, aber kein Ver- } \\
\text { lust der Abduktion }\end{array}$ & Knoten ohne Winkeldefizit & $=0,5$ \\
\hline Späte RT-Indikation & $\mathrm{N} / \mathrm{l}$ & $\begin{array}{l}\text { Knoten, aber kein Ver- } \\
\text { lust der Abduktion }\end{array}$ & $\begin{array}{l}\text { Beginnendes Winkeldefizit } \\
\text { der Finger } \\
10^{\circ}\left(-30^{\circ}\right)\end{array}$ & $=0,5$ \\
\hline $\begin{array}{l}\text { Keine RT-Indikation } \\
\rightarrow \text { operative } \\
\text { Methoden }\end{array}$ & I & $\begin{array}{l}\text { Abduktionswinkel } \\
45-30^{\circ}\end{array}$ & $\begin{array}{l}\text { Winkeldefizit der Finger } \\
11-45^{\circ}\end{array}$ & $=1$ \\
\hline $\begin{array}{l}\text { Operation, wenn } \\
\text { notwendig }\end{array}$ & II & $\begin{array}{l}\text { Abduktionswinkel } \\
29-15^{\circ}\end{array}$ & $\begin{array}{l}\text { Winkeldefizit der Finger } \\
46-90^{\circ}\end{array}$ & $=2$ \\
\hline $\begin{array}{l}\text { Operation, wenn } \\
\text { notwendig }\end{array}$ & III & $\begin{array}{l}\text { Abduktionswinkel } \\
14-0^{\circ}\end{array}$ & $\begin{array}{l}\text { Winkeldefizit der Finger } \\
91-135^{\circ}\end{array}$ & $=3$ \\
\hline & IV & Nicht definiert & $\begin{array}{l}\text { Winkeldefizit der Finger } \\
\geq 135^{\circ}\end{array}$ & $=4$ \\
\hline $\begin{array}{l}\text { Individuelle } \\
\text { Therapie }\end{array}$ & $\mathrm{R}$ & \multicolumn{3}{|c|}{$\begin{array}{l}\text { Jedes progrediente Stadium nach stattgehabter } \\
\text { Operation }\end{array}$} \\
\hline Tubiana-Score & & Maximal 3 Punkte & Maximal: 5-mal 4 Punkte & $=23$ \\
\hline
\end{tabular}

Tab. 2 Indikation zur Strahlentherapie (RT) und Klassifikation des Morbus Ledderhose

\begin{tabular}{|c|c|c|}
\hline RT-Indikation & Stadium & Definition \\
\hline Keine RT-Indikation & I- unifokaler Befall & $\begin{array}{l}\text { Fokale (isolierte) Erkrankung beschränkt auf eine } \\
\text { kleine Fläche der Fußfaszie. Keine Haftung an der } \\
\text { Haut, kein tiefes Eindringen in den Muskelbereich } \\
\text { (Plantaraponeurose) }\end{array}$ \\
\hline Frühe RT-Indikation & II - multifokaler Befall & $\begin{array}{l}\text { Multifokale Erkrankung, mit oder ohne Ausbrei- } \\
\text { tung (distal oder proximal). Keine Haftung an der } \\
\text { Haut, kein tiefes Eindringen in den Muskelbereich } \\
\text { (Plantaraponeurose) }\end{array}$ \\
\hline Frühe RT-Indikation & $\begin{array}{l}\text { III - Stadium II plus } \\
\text { tiefe Erweiterung in } \\
\text { eine Richtung }\end{array}$ & $\begin{array}{l}\text { Multifokale Erkrankung, mit oder ohne Ausbrei- } \\
\text { tung. Entweder Haftung an der Haut (III A) oder } \\
\text { tiefes Eindringen in den Muskelbereich (III B) }\end{array}$ \\
\hline Späte RT-Indikation & $\begin{array}{l}\text { IV - Stadium II plus } \\
\text { tiefe Erweiterung in } \\
\text { beide Richtungen }\end{array}$ & $\begin{array}{l}\text { Multifokale Erkrankung, mit oder ohne Ausbrei- } \\
\text { tung. Sowoh/ Haftung an der Haut als auch tiefes } \\
\text { Eindringen in den Muskelbereich }\end{array}$ \\
\hline Individuelle Therapie & Rezidiv & $\begin{array}{l}\text { Jeglicher Progress nach chirurgischer Interven- } \\
\text { tion }\end{array}$ \\
\hline $\begin{array}{l}\text { Andere Parameter zur } \\
\text { Beschreibung }\end{array}$ & $\begin{array}{l}\text { Spezielle Symbole } \\
\text { und Funktionen }\end{array}$ & 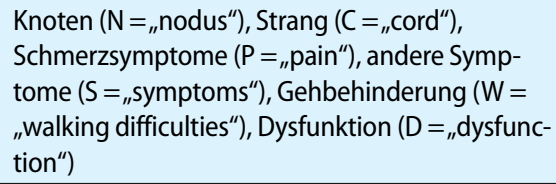 \\
\hline
\end{tabular}

Meist wird in der frühen MD/MLPhase eine Wait-and-see-Strategie empfohlen, da noch keine konservative Behandlung fest etabliert werden konnte. Glukokortikoidinjektionen können zwar zur Regression führen, aber auch schwere Komplikationen, beispielsweise Atrophie an der Injektionsstelle oder Ruptur der Sehnen, induzieren. Außerdem kann damit keine langfristige Auswirkung auf den Krankheitsverlauf erzielt werden [8]. Die findliche proliferierende Fibroblasten und Myofibroblasten.

Ionisierende Strahlen reduzieren deren proliferative Aktivität und induzieren die Bildung von freien Radikalen und die Reduzierung der Zelldichte [11]. In den späteren Krankheitsstadien, die durch Reparatur und Kontraktion von Bindegewebe gekennzeichnet sind, sind ionisierende Strahlen nicht mehr wirksam. Somit gilt prinzipiell, dass die Radiotherapie nur in den frühen Phasen des MD angewendet werden kann, um ein weiteres Fortschreiten der Krankheit und spätere Dysfunktionen oder gar die Notwendigkeit von operativen Maßnahmen zu vermeiden.

Dies erfordert vom Arzt immer eine sorgfältige körperliche Untersuchung sowohl von den Händen als auch den Füßen und, wenn möglich, eine Verlaufskontrolle über 3 bis 6 Monate, um Veränderungen und ein Voranschreiten der Krankheit früh zu erkennen. Beispielhaft zeigt - Abb. 3 die fortschreitende Entwicklung nach 1 Jahr Nachbeobachtung.

\section{Etablierte \\ Strahlentherapiekonzepte}

Bisher publizierte Studien zeigen, dass die Verwendung von 3 Gy Einzeldosis und eine Gesamtdosis von über $20 \mathrm{~Gy}$ zu nachhaltigen Erfolgen führt. Nur in wenigen Studien wurden verschiedene Dosiskonzepte vergleichend kontrolliert. Seegenschmiedt et al. [12] verglichen in einer randomisierten Studie (Essener Studie) eine Beobachtungsgruppe gegen 2 Strahlentherapiekonzepte: Konzept A = 7 Fraktionen à 3-21 Gy Gesamtdosis (Zeitraum 2 Wochen) oder Konzept $B=5$ Fraktionen à 3 Gy in 2 Serien bis 30 Gy Gesamtdosis (Behandlungszeitraum 3 Monate/2 Monate Pause zwischen den beiden Serien).

In beiden RT-Gruppen konnten eine signifikant höhere Vermeidung eines Krankheitsprogresses und die Vermeidung einer späteren chirurgischen Intervention im Vergleich zur Beobachtungsgruppe erzielt werden. 


\begin{tabular}{|c|c|c|c|c|c|}
\hline $\begin{array}{l}\text { Modifizierte Tubiana- } \\
\text { Stadieneinteilung } \\
\text { (Streckdefizit/Win- } \\
\text { kel/Handfunktion) }\end{array}$ & $\begin{array}{l}\mathrm{N} \\
0^{\circ} \\
\text { Progress an Knoten } \\
\text { und Strängen; Kein } \\
\text { Defizit }\end{array}$ & $\begin{array}{l}\mathrm{N} / \mathrm{I} \\
0^{\circ}-10^{\circ} \\
\text { Geringes Winkel- } \\
\text { defizit }\end{array}$ & $\begin{array}{l}\text { I } \\
10^{\circ}-45^{\circ} \\
\text { Zunehmendes Win- } \\
\text { keldefizit }\end{array}$ & $\begin{array}{l}\text { II } \\
46^{\circ}-90^{\circ} \\
\text { Mittelgradiges Win- } \\
\text { keldefizit }\end{array}$ & $\begin{array}{l}\text { III-IV } \\
90^{\circ} \\
\text { Deutliches Winkel- } \\
\text { defizit }\end{array}$ \\
\hline Wait-and-see-Strategie & $\begin{array}{l}\text { Ruhendes Stadium } \\
\text { ohne Progress }\end{array}$ & & & & \\
\hline Radiotherapie & $\begin{array}{l}\text { Progress der Knoten/ } \\
\text { Stränge in den letzten } \\
3 \text { bis } 6 \text { Monaten }\end{array}$ & $\begin{array}{l}\text { Progress } \pm \text { minimales } \\
\text { Funktionsdefizit }\end{array}$ & $\begin{array}{l}\text { Progress bis maximal } \\
30^{\circ} \text { Streckdefizit }\end{array}$ & - & (Post-OP) \\
\hline $\begin{array}{l}\text { Minimalinvasive } \\
\text { Chirurgie/Nadelfaszio- } \\
\text { tomie }\end{array}$ & - & - & $\begin{array}{l}\text { Progress nach } 30^{\circ} \\
\text { Defizit }\end{array}$ & $\begin{array}{l}\text { Progress nach } 45^{\circ} \\
\text { Defizit }\end{array}$ & $(+)$ \\
\hline Kollagenaseinjektion & - & - & $(+)$ & $\begin{array}{l}\text { Progress nach } 45^{\circ} \\
\text { Defizit }\end{array}$ & $(+)$ \\
\hline Handchirurgie & - & - & - & $\begin{array}{l}\text { Bei komplexer Situ- } \\
\text { ation }\end{array}$ & $\mathrm{Ja}$ \\
\hline
\end{tabular}

\section{Sorgfältige \\ Strahlentherapieplanung}

Das klinische Zielvolumen umschließt auf der palmaren Hautoberfläche alle Knoten und Stränge plus eine „Sicherheitszone“ von mindestens $1 \mathrm{~cm}$ nach lateral und eine Erweiterung um bis zu $2 \mathrm{~cm}$ nach proximal und distal. Die Bestrahlung erfolgt mit niederenergetischen Photonen (100$125 \mathrm{kV}$ ) am Orthovolt-Gerät oder niederenergetischen Elektronen (4 MV: Hände/6 MV: Füße) am Linearbeschleuniger. Die Referenzdosis wird in einer Tiefe von 0-10 mm je nach Dicke der Haut und Größe der Läsion an Hand oder Fuß berechnet. Die Zielvolumen werden mit Bleiabsorbern oder Bleigummi individualisiert. Die Abb. 4a, b, 5 und 6 zeigen typische Bestrahlungsfelder und die Durchführung der Bestrahlung am Linearbeschleuniger.

Bisher nicht etabliert ist die postoperative Strahlentherapie. Damit könnte ein frühes Rezidiv vermieden und ein längerfristiges stabiles Intervall erreicht werden. Bei diesen Fällen wird nicht nur der betroffene operierte Finger, sondern es werden auch die anderen Finger und die palmare Faszie behandelt (5). Die postoperative Bestrahlung ist die Ausnahme und sollte sorgfältig und individuell mit dem Operateur besprochen werden.

\section{Klinische Ergebnisse der Strahlentherapie}

Seit 50 Jahren konnte in über 20 klinischen Studien der Nutzen der Strahlentherapie bei Fibromatosen belegt werden. Inzwischen liegen auch prospektive klinische Studien mit großen Patientenkollektiven vor, die über eine lange Zeitspanne nachbeobachtet sind.

\section{Erlanger Studie}

Die retrospektive Studie aus Erlangen [13] wurde in den frühen 1980er-Jahren begonnen und erfasste 135 Patienten (mit 208 Händen) in den Jahren 1982 bis 1993 , die wegen eines MD mit 10-mal 3 Gy bis 30 Gy in 2 Serien zu je 15 Gy (5-mal 3 Gy) bestrahlt worden sind. Der Nachsorgezeitraum betrug median 13 Jahre. Dabei fand sich ein positiver Langzeiteffekt: 123 (59\%) der Hände blieben stabil, $20(10 \%)$ zeigten eine klinische Verbesserung, und bei 65 Patienten (31\%) fand sich ein Krankheitsprogress. Wichtigster prognostischer Faktor für das Therapienansprechen war das Stadium: Patienten mit Stadium $\mathrm{N}$ blieben stabil oder besserten sich in $87 \%$; im Stadium N/I war dies bei $70 \%$ der Fall. In den fortgeschrittenen Stadien I-IV kam es in den bestrahlten Arealen häufiger zum Progress (Stadium I: in $62 \%$, Stadium II: in $86 \%$ ). Insgesamt wurde bei $66 \%$ eine langfristige Symptomkontrolle erreicht. Bei insgesamt $31 \%$ der Fälle trat ein Krankheitsprogress auf: im RT-
Feld bei 14\%, außerhalb des RT-Feldes bei $3 \%$ und sowohl innerhalb und außerhalb des RT-Feldes bei $14 \%$.

In $32 \%$ der bestrahlten Handregionen traten geringe Spätnebenwirkungen (Grad 1) auf, meist eine leichte Hautdesquamation. Auch bei evtl. notwendiger Handchirurgie konnte keine erhöhte Komplikationsrate bei Patienten mit vorangegangener Strahlentherapie festgestellt werden. Bislang wurde bei keinem bestrahlten Fall ein Sekundärmalignom festgestellt.

\section{Essener Studie}

Eine kontrollierte klinische Studie aus Essen ging der Frage nach der optimalen Strahlendosis nach [14]. Insgesamt wurden 489 Patienten (718 Hände) behandelt und über mindestens 5 Jahre (Mittel 8,5 Jahre) nachbeobachtet und ausgewertet. Im Rahmen der Studie erfolgte nach der klinischen Untersuchung zunächst je nach Wunsch des Patienten eine Einteilung in die Wait-and-see- (Beobachtung ohne Bestrahlung) oder die Strahlentherapiegruppe: Für die klinische Beobachtung („wait and see“) entscheiden sich 83 Patienten (122 Hände), für die Strahlentherapie (RT) 406 Patienten (596 Hände). Im Falle der Strahlentherapie erfolgte eine Randomisierung in 2 Gruppen:

- Gruppe A (207 Patienten/303 Hände) erhielt 10-mal 3 Gy (Gesamt: 30 Gy) in 2 Serien, 5-mal 3 Gy mit einer Pause von mindestens 12 Wochen. 


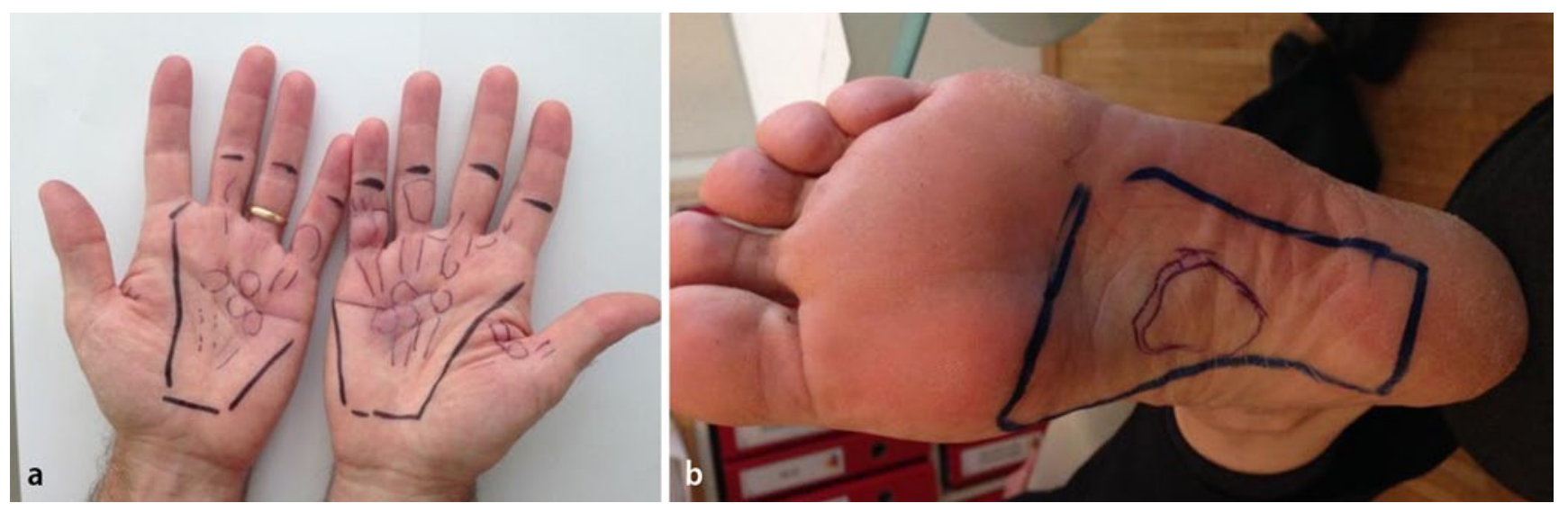

Abb. $4 \Delta$ Behandlungsfelder a bei Morbus Dupuytren beidseits und b Morbus Ledderhose links (mit freundl. Genehmigung von Prof. Dr. M.H. Seegenschmiedt). a Dicke Linie: umfasst das Strahlentherapie (RT)-Feld mit der betroffenen palmaren Handregion plus Sicherheitszone. Kreise markieren die genaue Lage der Knoten. Striche markieren die Lage der Strangbildungen. Die Daumenregion wird meist aus dem RT-Feld ausgeschlossen (bei geringem Funktionsverlust). b Dicke Linie: RT-Feld umfasst den zentralen, palpablen Knoten und eine Sicherheitszone von mindestens $1 \mathrm{~cm}$. Zone zwischen Vorfußballen und Ferse $($ hier $12 \times 7 \mathrm{~cm}$ )
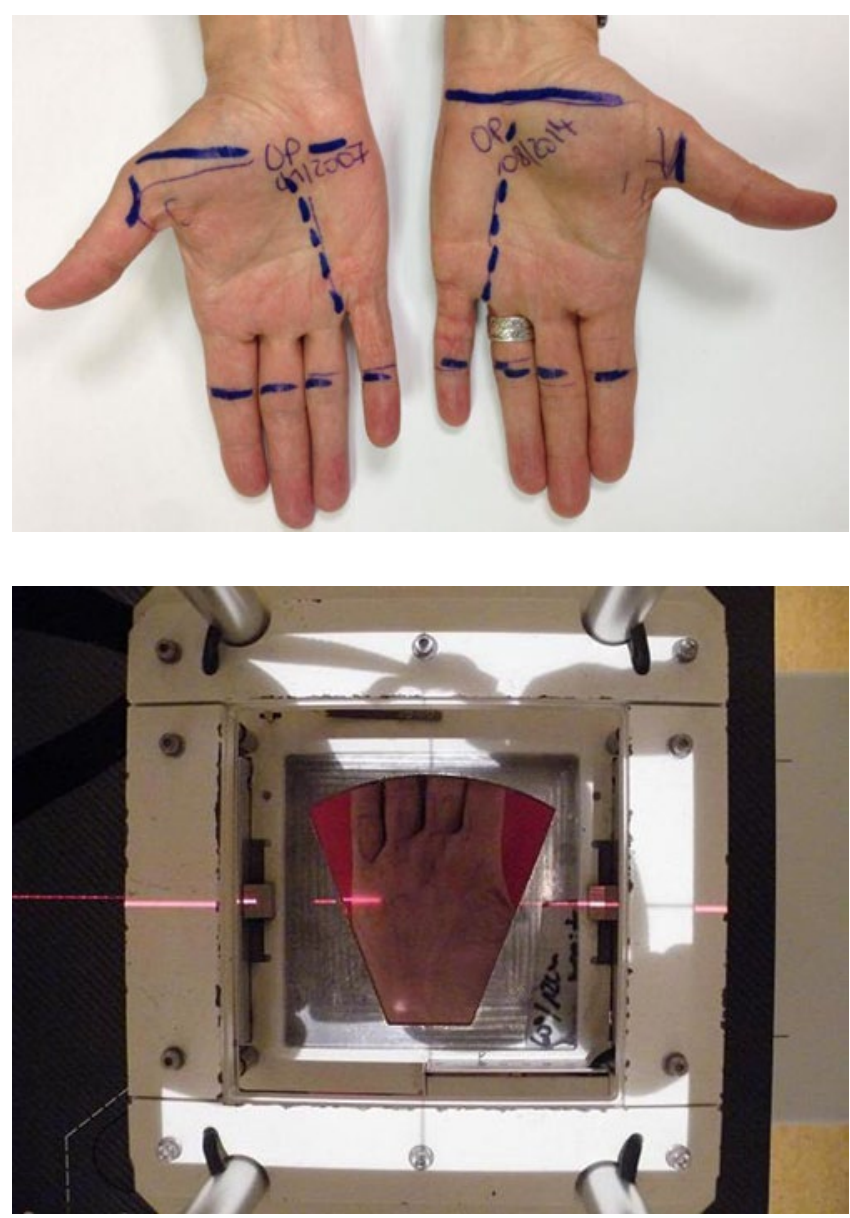

Abb. $5<$ Behandlungsfelder bei beidseitigem Morbus Dupuytren nach Handchirurgie wegen Progress. (Mit freundl. Genehmigung von Prof. Dr. M.H. Seegenschmiedt)

Abb. $6<$ Behandlungsfeld (Beam's Eye View, Sicht aus Linearbeschleunigergerät) auf den Elektronentubus mit individuell angeformten Bleilamellen. (Mit freundl. Genehmigung von Prof. Dr. M.H. Seegenschmiedt)

- Gruppe B (199 Patienten/297 Hände) erhielt 7-mal 3 Gy (Gesamt: 21 Gy) in 1 Serie über einen 2-wöchigen Behandlungszeitraum (3-mal RT-Anwendung pro Woche).
Unterschiede bei den relevanten Patienten- und Krankheitsparametern.

Die Nachkontrollen erfolgten nach 3 und 12 Monaten und im Langzeitverlauf nach 5 Jahren und zum Ende der gesamten Auswertungsperiode. Insgesamt zeigte sich bei 119 Händen aus der RT-Gruppe $(16,5 \%)$ eine objektive Remission der Knoten und/oder Stränge, bei 383 (53\%) Händen wurde ein stabiler Zustand erzielt. Bei 206 Fällen (29\%) wurde ein klinischer Progress verzeichnet, worauf sich 97 Patienten (13,5\%) im weiteren Verlauf einem operativen Eingriff [Handchirurgie (H-CX)] unterziehen mussten.

Die Progressionsrate („progressive disease“, PD) lag in der unbehandelten Kontrollgruppe signifikant höher (jeglicher Progress in $62 \%$, H-CX bei $30 \%$ ) als in den beiden RT-Gruppen (21 Gy: PD $24 \% / \mathrm{H}-\mathrm{CX}$ in $12 \%$; 30 Gy: PD 19,5\%/HCX in $8 \% ;(p<0,0001)$. In der RT-Gruppe traten insgesamt bei 50 (8\%) Händen Rezidive/Progress im RT-Feld und 114(19\%) außerhalb des RT-Feldes auf. Eine nachfolgende Handchirurgie wurde in diesen Fällen ohne erhöhte Morbidität auch nach erfolgter RT durchgeführt werden.

Die Nebenwirkungsrate entsprach dem Erlanger Kollektiv (akute Nebenwirkungen nach CTC Grad 1: 26,5\%, Grad 2: $2,5 \%)$. Bei $14 \%$ traten Spätnebenwirkung Grad 1 auf. Bisher traten keine Karzinome im Beobachtungszeitraum auf. Uni- und multivariate Prognosefaktoren für das Auftreten einer Progression wa- 
ren: Nikotin, Symptome vor der RT länger als 24 Monate, das Dupuytren-Stadium, ein Streckdefizit und die Fingerbeteiligung $(p<0,05)$. Wichtigster unabhängiger prognostischer Faktor war die Strahlentherapie.

Beide RT-Gruppen waren der Beobachtungsgruppe damit hoch überlegen, während die Unterschiede zwischen den beiden RT-Serien nach 5 Jahren zwar unterschiedlich, aber noch nicht signifikant waren. Ansonsten erhöhte eine vorangegangene RT nicht die Komplikationsrate im Fall einer im Verlauf notwendigen handchirurgischen Operation.

\section{Ledderhose-Studie}

Zum ML liegen bislang nur wenige klinische Studien vor, die heterogene Kollektive erfassen. Die größte Serie aus Essen [15] untersuchte Langzeitresultate bei 91 Patienten (136 Füße), die progrediente Knoten und Stränge vor Beginn der Strahlentherapie hatten. Bei 88 (97\%) bestanden zusätzlich Symptome wie Taubheit, Schmerzen u. a., bei 86 (95\%) schmerzbedingte Gangstörungen. Bei 35 Füßen lag ein Progress nach Fußchirurgie vor; 67 Patienten (134 nichtbetroffene Füße) dienten als Kontrollgruppe ohne RT.

Die Bestrahlung erfolgte am Orthovolt-Gerät (125-150 kV Photonen) mit 10-mal 3-30 Gy in 2 Serien bis 30 Gy Gesamtdosis. Bei 60 Füßen (44\%) stabilisierte sich der Befund, bei 65 (48\%) kam es zum partiellen Ansprechen mit Verkleinerung der Knoten und/oder der Stränge und/oder der Symptome. Bei 35 der 65 Fälle entwickelte sich sogar eine komplette Remission von Schmerzen mit Rückbildung der Knoten/Stränge. Symptome und Funktionsstörungen besserten sich durch die Bestrahlung in bis zu $90 \%$. Die Patientenzufriedenheit verbesserte sich bei 81 (89\%). Ein Progress nach RT trat nur bei 6 Patienten (11 Füße) auf. Eine Salvage-Operation erfolgte bei 5 von 6 Patienten (d.h. an 9 der 11 Füße). Patienten in der Kontrollgruppe ohne Bestrahlung hatten eine höhere Progressions- und Operationsrate.

Akute Strahlenreaktionen traten in $21 \%$ auf (Grad 1: leichte Hautrötung/ Grad 2: 5\%). Späte Nebenwirkungen (LENT Grad 1: geringe Hauttrocken- heit oder Fibrose) wurden bei 22 Patienten $(16 \%)$ beobachtet. Akut- oder späte Reaktionen Grad 3 wurden nicht beobachtet.

In einer anderen retrospektiven Studie [16] wurden 2 RT-Schemata (10-mal 3 Gy oder 8-mal 4 Gy) und verschiedene RT-Techniken verglichen, die aber keine Unterschiede im Ergebnis aufwiesen. Zwei Jahre nach RT wurden weder ein Progress noch eine Verschlechterung der Symptome beobachtet. Eine komplette Remission wurde bei $33 \%$, eine partielle Remission (reduzierte Anzahl und Größe) bei $55 \%$ festgestellt; bei $12 \%$ traten keine Veränderungen auf. Darüber hinaus besserten sich die Schmerzen in $63 \%$, das Gangbild in $73 \%$ und die Patientenzufriedenheit in $92 \%$.

\section{Ausblick: kombinierte Konzepte (bimodale Behandlung)}

In Zukunft könnte die Anwendung der RT auch postoperativ nach Fuß- oder Handchirurgie in kontrollierten klinischen Studien untersucht werden, um durch postoperative RT die frühen Rezidive nach minimalinvasiver und/oder klassischer Chirurgie zu verhindern. Bislang liegen für den MD keine und für den ML nur wenige Studien mit geringer Evidenz vor.

\section{Fazit für die Praxis}

- Die Radiotherapie ist inzwischen eine etablierte und effektive Therapieoption für bestimmte hyperproliferative Erkrankungen und gutartige Neubildungen (S2-Leitlinie).

- In frühen Stadien des MD/ML bietet sie eine effektive Prophylaxe mit akzeptabler Akut- und Spättoxizität im Langzeitverlauf. Mit einer Dosis von 30 Gy scheint die chirurgische Komplikationsrate nicht erhöht. Somit empfehlen wir, die $\mathrm{RT}$ routinemäßig in den frühen Stadien des MD/ML als nichtinvasive Therapieoption innerhalb der ersten 1 bis 2 Jahre nach Diagnose und Progress durchzuführen.

- Das etablierte und klinisch meist geprüfte Radiotherapiekonzept besteht aus 10 Fraktionen à $3 \mathrm{~Gy}$ Einzeldosis in 2 Serien von je $15 \mathrm{~Gy}$ innerhalb von 3 Monaten.
- Prospektive Studien mit Vergleich der RT zu minimalen oder offenen Operationstechniken oder als kombinierte Behandlung stehen noch aus. Auch die Berücksichtigung bei nationalen und internationalen Leitlinien ist erforderlich.

- Eine nationale DEGRO-S2e-Konsensus-Leitlinienempfehlung für zahlreiche hyperproliferative Erkrankungen und gutartige Neubildungen existiert seit 2015 [17]. Allerdings wird auch bei 2 aktuellen Reviews [18, 19] unter den aufgezählten Therapieverfahren (Kollageninjektion, Chirurgie, Schockwellentherapie) die Strahlentherapie nicht erwähnt - ein Umstand, der angesichts wachsender Evidenz in Zukunft zu ändern sein wird.

\section{Korrespondenzadresse}

\section{K. Piefel}

Strahlenzentrum Hamburg

Langenhorner Chaussee 369, 22419 Hamburg

karen.piefel@googlemail.com

\section{Einhaltung ethischer Richtlinien}

Interessenkonflikt. K. Piefel, T. Schneider und M.H. Seegenschmiedt geben an, dass kein Interessenkonflikt besteht.

Dieser Beitrag beinhaltet keine Studien an Menschen oder Tieren.

The supplement containing this article is not sponsored by industry.

\section{Literatur}

1. Enzinger FM, Weiss SW (1995) Soft tissue tumors, 3. Aufl. CRC Press, St. Louis. (ISBN 10: 0815131321 ISBN 13:9780815131328)

2. Rodemann HP, Bamberg M (1995) Cellular basis of radiation-induced fibrosis. Radiother. Oncol. 35:83-90

3. Millesi H (1981) Dupuytren-Kontraktur. In: Nigst $H$, Buck-Gramcko D, Millesi H (Hrsg) Hand-Chirurgie, Bd I. Thieme, Stuttgart, S 1500-1557

4. Donato RR, Morrison WA (1996) Dupuytren's disease in the feet causing flexion contractures in the toes. J Hand Surg Br 21(3):364-366

5. Strickland JW, Idler RS, Creighton JC (1990) Dupuytren's disease. Indiana Med 83(6):408-409

6. Tubiana R, Michon J, Thomine JM (1966) Evaluation chiffree des deformations dans la maladie de Dupuytren. In: Maladie du Dupuytren (monographies du G.E.M.). Expansion Scientifique Francaise, Paris

7. Sammarco GJ (2001) Osteotomy of the foot and ankle. Foot Ankle Clin 6(3):xi-xiii 
8. Ketchnum LD, Donahue TK (2000) The injection of nodules of Dupuytren's Disease with triamcinolone acetonide. J Hand Surg 25:1157-1162

9. Keilholz L, Seegenschmiedt MH, Sauer R (1996) Radiotherapy for prevention of disease progression in early-stage Dupuytren's con-tracture: initial and long-term results. Int J Radiat Oncol Biol Phys 36(4):891-897

10. Heyd R, Dorn AP, Herkströter M, Rödel C, MüllerSchimpfle M, Fraunholz I (2010) Radiation therapy for early stages of Morbus Led-derhose. Strahlenther Onkol 186(1):24-29

11. Murrell GAC, Francis MJO (1994) Oxygen free radicals and Dupuytren's disease. In: Berger A, Delbrück A, Brenner P, Hinzmann R (Hrsg) Dupuytren's disease. Springer, Berlin, S 227-234

12. Seegenschmiedt MH, Micke $O$ (2012) Radiotherapy of non-malignant diseases. Post, present and future. Strahlenther Onkol 188(Suppl 3):272-290

13. Betz N, Ott OJ, Adamietz B, Sauer R, Fietkau R, Keilholz L (2010) Radiotherapy in early-stage Dupuytren's contracture. Long-term results after 13 years. Strahlenther Onkol 186(2):82-90

14. Seegenschmiedt MH, Keilholz L, Wielpütz M, Schubert C, Fehlauer F (2012a) Long-term outcome of radiotherapy for early stage Dupuytren's disease: a phase iii clinical study. In: Eaton C, Seegenschmiedt MH, Bayat A, Gabbiani G, Werker P, Wach W (Hrsg) Dupuytren's disease and related hyperproliferative disorders - principles, research, and clinical perspectives. Springer Publishers, Heidelberg, S 349-371. http://link.springer.com/chapter/ 10.1007\%2F978-3-642-22697-7_44

15. Seegenschmiedt MH, Wielpütz $M$, Hanslian E, Fehlauer $F$ (2012b) Long-term outcome of radiotherapy for early stage Dupuytren's disease: a phase III clinical study. In: Eaton C, Seegenschmiedt MH, Bayat A, Gabbiani G, Werker P, Wach W (Hrsg) Dupuytren's disease and related hyperproliferative disorders - principles, research, and clinical perspectives. Springer Publishers, Heidelberg, S 409-427. http://link.springer.com/chapter/10.1007/978-3-642-22697-7_50

16. Heyd R, Dorn AP, Herkströter M, Rödel C, MüllerSchimpfle M, Fraunholz I (2010) Radiation therapy for early stages of Morbus Ledderhose. Strahlenther Onkol 186(1):24-29

17. Seegenschmiedt MH, Micke $O$, Niewald M, Mücke R, Eich HT, Kriz J, Heyd R, German Cooperative Group on Radiotherapy of Benign Diseases (GCGBD) (2015) DEGRO guidelines for the radiotherapy of non-malignant disorders: Part III: Hyperproliferative disorders. Strahlenther Onkol 191(7):541548 (Epub 2015 Mar 10)

18. van Dijk D, Finigan P, Gerber RA, Szczypa PP, Werker PM (2013) Recognition, diagnosis and referral of patients with Dupuytren's disease: a review of current concepts for general practitioners in Europe. Curr Med Res Opin 29(3):269-277. doi:10.1185 /03007995.2013.766163

19. Mafi R et al (2012) recent surgical and medical advances in the treatment of Dupuytren's disease - a systematic review of the literature. Open Orthop J 6:77-82. doi 10.2174/1874325001206010077 